This item was submitted to Loughborough's Research Repository by the author.

Items in Figshare are protected by copyright, with all rights reserved, unless otherwise indicated.

\title{
Syntax and sequencing of assembly instructions
}

PLEASE CITE THE PUBLISHED VERSION

https://www.crcpress.com/Advances-in-Usability-Evaluation-Part-II/Rebelo-Soares/p/book/9781466560543

PUBLISHER

CRC Press (@ Taylor \& Francis Group)

VERSION

AM (Accepted Manuscript)

PUBLISHER STATEMENT

This work is made available according to the conditions of the Creative Commons Attribution-NonCommercialNoDerivatives 4.0 International (CC BY-NC-ND 4.0) licence. Full details of this licence are available at: https://creativecommons.org/licenses/by-nc-nd/4.0/

\section{LICENCE}

CC BY-NC-ND 4.0

\section{REPOSITORY RECORD}

Thorvald, Peter, Gunnar Backstrand, Dan Hogberg, and Keith Case. 2019. "Syntax and Sequencing of Assembly Instructions". figshare. https://hdl.handle.net/2134/34396. 
In F. Rebelo and M. M. Soares (Eds.), 'Advances in Usability Evaluation Part II',

Proceedings of the Fourth International Conference on Applied Human Factors and Ergonomics, AHFE 2012 (pp. 266-275). San Francisco, USA, 21-25 July, 2012: CRC Press.

\title{
Syntax and Sequencing of Assembly Instructions
}

\author{
Peter Thorvald, Gunnar Bäckstrand, Dan Högberg \& Keith Case \\ University of Skövde \\ Skövde, Sweden \\ peter.thorvald@his.se \\ Swerea IVF \\ Stockholm, Sweden \\ Loughborough University \\ Loughborough, UK
}

\begin{abstract}
Minimalism of design is a concept often found in Human-computer interaction (HCI). It is a concept that emphasizes the presentation of as little information as possible to reduce the perceptual strain and visual search of the subject. However, in a manufacturing context, such as in manual assembly, state of the art information presentation is rarely minimalistic. Rather, organizations tend to push out as much information as possible without necessarily concerning themselves with how this information is presented to, or perceived by, the worker. This leads to a situation that is far from ideal from an HCI perspective, likely to reduce human performance and wellbeing, in turn negatively affecting overall production system performance. Obviously, there are several potential ways of addressing this issue. Perhaps the most evident way is to simply reduce the amount of information that is presented and only present the essentials. This paper will investigate and discuss how information presentation can be minimized without reducing the information content through information syntax and layout.
\end{abstract}

\section{INTRODUCTION}

This paper will investigate the potential use of alternate syntax and alternate sequencing of data in information presentation for manual assembly. Two 
hypotheses have been formulated based on previous work as well as experience from the automotive assembly industry (Bäckstrand, 2010; Thorvald, 2011):

Hypothesis 1 Using unsequenced data and thus minimizing the amount of presented information reduces errors and assembly time.

Hypothesis 2 The use of symbols as opposed to article numbers reduces errors and assembly time.

\section{Hypothesis 1}

In the investigation of using sequenced and unsequenced information, hypothesis 1 suggests that using unsequenced, batched information as opposed to sequenced information reduces assembly time (productivity) and errors (quality). This is argued to be due to the reduction of data presented to the worker.

\begin{tabular}{|l|c|l|}
\hline Part & Quantity & Description \\
\hline Part A & 1 & Assemble part \\
\hline Part B & 1 & Assemble part \\
\hline Part A & 1 & Assemble part \\
\hline
\end{tabular}

Figure 1. Sequenced information presentation.

As can be seen in Figure 1, sequenced information presentation presents parts in the order that they are to be assembled. While this might be preferred when presenting information to novices, such as when assembling furniture from IKEA, the expertise in a manufacturing assembly environment can be expected to be higher. Consequently, this research proposes and explores the use of unsequenced, batched information presentation so that the information presented in Figure 1 may look like Figure 2:

\begin{tabular}{|l|c|l|}
\hline Part & Quantity & Description \\
\hline Part A & 2 & Assemble part \\
\hline Part B & 1 & Assemble part \\
\hline
\end{tabular}

Figure 2. Unsequenced, batched information presentation.

It is argued that presenting unsequenced, batched information as opposed to sequenced information saves space on the information medium, reduces cognitive strain related to information search, and saves in the non value adding activity of information search (Sawhney et al., 2009). Naturally, simple cases like the ones in the figures above, where the reduction of data is limited to the elimination of one row, would not be considered a major improvement but applying this to normal work instructions with 15-20 different parts might induce an improvement in both quality and productivity.

\section{Hypothesis 2}


The suggestion of using symbols as opposed to article numbers in information presentation for assembly is based on the idea that symbols carry semantic content about themselves that article numbers typically do not, and therefore they are easier to percept, process and recall. It is evident that it is most likely to be easier to process symbol representation over article numbers as the symbols are shorter, consisting of one character, whereas article numbers usually consist of several characters. However, as mentioned above, the number of characters within a representing element is not argued to be the reason for this difference. Rather, any difference found between the two is believed to be a result of the semantic content that the two representational modes include. An article number, "564163", has no connection to the long term memory of the user and is therefore subject to the risk of short term memory limitations. A symbol, ' $\Omega$ ', on the other hand, is most likely established in the user's long term memory as "Omega". It is very likely to have personal meaning to the user, for example, the user might associate it to Greece and the Greek alphabet, it might be associated to electrical engineering a $\Omega$ is used as the symbol for ohm, a unit of electrical resistance, etc. The associative possibilities are great and this is believed to result in better recognition, recall and matching with the same symbol on the parts shelf.

Symbols are recognized in long term memory and are easily available for recognition, recall and memory. Article numbers are not connected to long term memory and thus will need to be kept in short term memory while the task is carried out.

\section{Experimental design}

To test hypotheses 1 and 2, an independent design experiment was set up where actual assembly workers assembled simple components according to different types of assembly information. All participants were given the same tasks but with different syntax to the information sources. The different modes of information design (states of the independent variable) were:

- Sequenced data presented with article numbers $\left(\mathrm{IV}_{1}\right)$

- Unsequenced data presented with article numbers $\left(\mathrm{IV}_{2}\right)$

- Unsequenced data presented with symbols $\left(\mathrm{IV}_{3}\right)$

Through the different states of the independent variable defined above, the hypotheses can be rejected or confirmed through the following analyses:

Comparisons between independent variable states $\mathrm{IV}_{1}$ and $\mathrm{IV}_{2}$ will confirm or reject hypothesis 1

- Comparisons between $\mathrm{IV}_{2}$ and $\mathrm{IV}_{3}$ will confirm or reject hypothesis 2

The risk of a confounding of the data appears when there are multiple potential sources for differences between test groups. This is why $\mathrm{IV}_{1}$ and $\mathrm{IV}_{3}$ cannot be compared, because they differ in two instances (Sequenced-unsequenced and article 
number-symbol). A comparison of these two would be meaningless as it is impossible to say what the result was caused by. However, $\mathrm{IV}_{1}$ can be compared with $\mathrm{IV}_{2}$ and $\mathrm{IV}_{2}$ can in turn be compared to $\mathrm{IV}_{3}$ since, in these comparisons the independent variables only differ in one instance for each pair.

\section{METHOD}

A total of 30 participants, randomly assigned to three independent groups took part in the study. All participants were experienced assembly workers with an age range of 20-42 years old. All participants had at least 2 years of experience in engine assembly and they were also deemed highly skilled assembly workers by Volvo Powertrain who loaned them to the study. Approximately $17 \%$ of the participants were female, a number that corresponds fairly accurately with gender diversity in the automotive assembly industry. The three states of the independent variable were tested on three separate days following each other. Variable state 1 was tested on day one, variable state 2 on day two and variable state 3 on day three. Ten subjects were assigned to each day.

Before the test, the subjects were briefed on how the test was to be carried out and how the assembly was to be completed. The task was to produce a predetermined pattern of LEGO ${ }^{\circledR}$ pieces with varying colours. Subjects were educated on the final shape of the product and informed that this would always be the same. Only the colours of the independent LEGO ${ }^{8}$ pieces would differ. Figure 3 shows the final shape of the finished product.

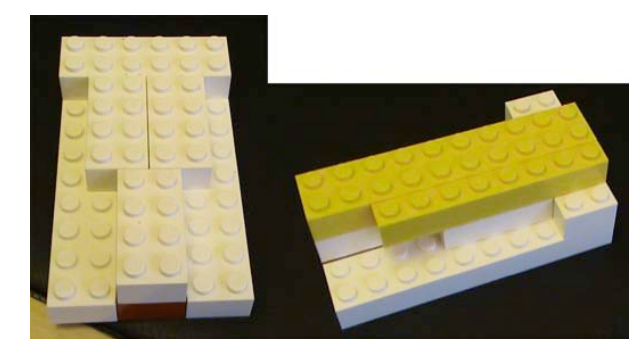

Figure 3. The final shape of the finished product shown in two states for clarity.

Figure 4, shows the building instructions that the subjects were presented with before the actual testing took place. Subjects were also offered a few pieces to testbuild the final product as part of the training. The building instructions were not available to the subjects during the test; only product information was available at that point. 

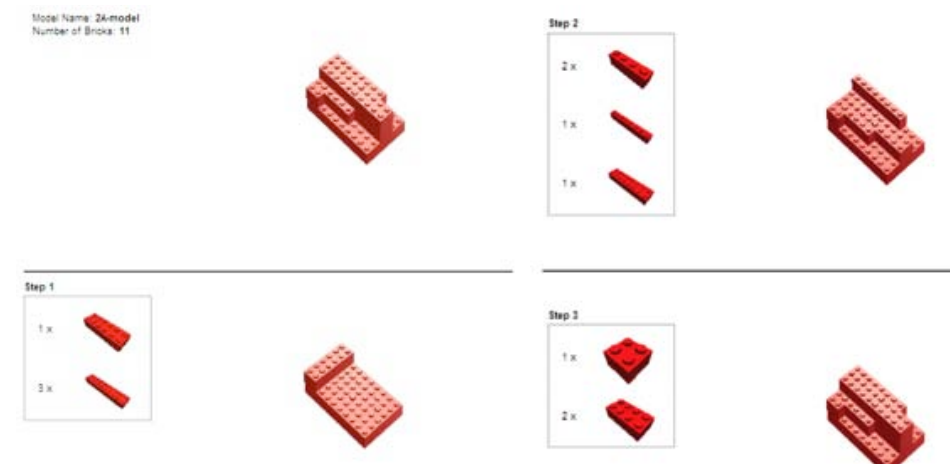

Figure 4. Building instructions. Top left frame is a description of the finished product. Each of the following frames describes the required parts and how they fit together.

\section{Equipment}

The test was carried out in a closed laboratory with the subject and two researchers present in the room. The task was carried out on a standing-height table with a material rack directly in front of it with material within reach of the subject. Information was presented to the subject via a computer laptop, placed on the table to the left of the subject and in front of the material rack used to supply LEGO ${ }^{\circledR}$ pieces to the experiment. The rack contained 13 boxes with different types of LEGO ${ }^{\circledR}$ pieces in them. Each type of LEGO ${ }^{\circledR}$ piece was also given a unique article number and a symbol. Article numbers and symbols were presented on the front edge of the material rack in relation to the boxes.

Three sets of instructions, based on the three states of the independent variable were used during the experiment. The instructions were identical in that they described the exact same sequence of the parts to be assembled, i.e. they contained the same information. The only thing that differed between the instructions was the way the product information was presented. For $\mathrm{IV}_{1}$, tested on day 1 , the assembly instructions were sequenced so that the parts of the product that were to be assembled first were presented first. Multiple quantities of articles only appeared in this state if the two articles were assembled together. The articles were presented through an article number and the quantity of the piece to be picked and assembled.

The sequence of products to be assembled was the same for all IV states and was divided into one high volume product ( $76 \%$ of the products) and six variants where a few of the pieces were exchanged from the high volume product. Each variant type had a frequency of about $4-5 \%$ with a total variant frequency of $24 \%$. The variants and high volume products were randomly distributed in the sequence of assembly. Figure 5 shows the actual assembly instructions for $\mathrm{IV}_{1}$. 


\begin{tabular}{|l|l|l|}
\hline \multicolumn{1}{|r|}{ Rubrik } & Antal & Instrukionstext \\
\hline - 56109282 & 2 & \\
- 55724016 & 2 & \\
- 56109282 & 1 & \\
- 57046203 & 1 & \\
- 55724016 & 1 & \\
- 57046203 & 1 & \\
- 60668495 & 1 & \\
- 56109282 & 1 & \\
\hline
\end{tabular}

Figure 5. Actual assembly information as presented to the s ubjects on day 1. Headlines were presented in Swedish and read from left to right; Headline, Quantity and Description.

On day $2, \mathrm{IV}_{2}$ was tested and the subjects were presented with unsequenced assembly information as can be seen in Figure 6. The information value and the syntax is still the same as in day 1 but the layout and the sequencing of the products has been manipulated.

\begin{tabular}{|l|l|l|}
\hline Rubrix & Antal & Instrictonstext \\
\hline - 56109282 & 4 & \\
- 57046203 & 2 & \\
- 55724016 & 3 & \\
- 60668495 & 1 & \\
& & \\
& & \\
& & \\
& & \\
& & \\
\end{tabular}

Figure 6. Unsequenced assembly instructions as presented on day 2.

On day 3 , testing of $\mathrm{IV}_{3}$ included the use of unsequenced symbols as opposed to day 2's use of unsequenced article numbers (Figure 7)

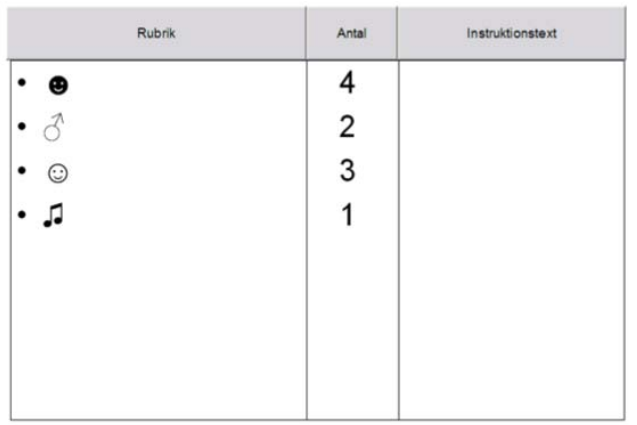

Figure 7. Assembly information through symbols as used on day 3. 


\section{Implementation}

The subjects were asked to finish as many assemblies as possible within 20 minutes. As all the subjects were experienced assembly workers they were asked to work at a normal work pace and try to assemble each product as well as possible. The number of products each subject was able to finish within the 20 minute limit varied from around 10 to 30 assemblies and for the analysis a mean for each subject was calculated. This way each group has 10 computable measurements.

\section{RESULTS}

An analysis of the difference between volume and variant products was made for all states of the independent variables. Surprisingly, only $\mathrm{IV}_{2}$ showed a significant difference between these suggesting that the sequencing of information has greater impact than the choice of syntax $(p=0,012)$.

So, in fact, only variable state 2 showed a significant difference between variant and volume products, and actually, for $\mathrm{IV}_{1}$, variants were assembled faster than volume products according to the means (volume $=53,17 \mathrm{~s}$, variants $=52,01 \mathrm{~s}$ ). However, since the result is not significant $(p=0,81)$ variants and volume products are statistically the same. Still, the indication that volume and variant products take the same time to assemble is very surprising as variants are generally believed to require more time than volume products and since the results are scattered between all IV's, a conclusion of this cannot be made. However, it could be argued that $\mathrm{IV}_{2}$ and $\mathrm{IV}_{3}$ both hit a floor effect in productivity in the sense that neither volume nor variant products could be assembled faster than around 25-30 seconds where means for both groups were found.

\section{Result of hypothesis 1}

The first hypothesis of the experiment was as follows:

- Using unsequenced data and thus minimizing the amount of presented information reduces errors and assembly time.

To test the above hypothesis, two sets of information sources were created. One where sequenced information was presented and one where the information was unsequenced and parts were batched together with other parts of the same kind.

Analysis of the means shows a difference between the groups and also a notably large effect size $(E S=0,9)$, which is probably the reason why a significant difference between the groups could be found despite the rather small sample size $(p=0,01)$.

Table 1 shows the descriptive statistics for the comparisons of $\operatorname{IV}_{1}$ and $\mathrm{IV}_{2}$. Both through simple analysis of the means and further significance analysis, it becomes evident that using unsequenced information presentation significantly improves productivity in manual assembly. 
Table 1. Descriptive statistics for comparison between $\mathrm{IV}_{1}$ and $\mathrm{IV}_{2}$

\begin{tabular}{|l|r|r|r|r|r|}
\hline & $\mathrm{N}$ & Minimum & Maximum & Mean & Std. Deviation \\
\hline $\mathrm{IV}_{1}$ & 10 & 28,56 & 96,78 & 52,5907 & 16,26455 \\
$\mathrm{IV}_{2}$ & 10 & 28,21 & 43,80 & 35,6690 & 4,51559 \\
Valid N (listwise) & 20 & & & & \\
\hline
\end{tabular}

\section{Result of hypothesis 2}

The second hypothesis of the experiment was as follows:

- Using symbols as opposed to traditional article numbers reduces errors and assembly time.

Just as the quality parameter arguably hit a floor effect due to the task being too easy, the productivity parameter for hypothesis 2 might have done the same. Analysis of the means in Table 2 shows a slight difference but deeper analysis showed no significant difference between the two groups $(p=0,231)$.

Table 2. Descriptive statistics for $\mathrm{IV}_{2}$ and $\mathrm{IV}_{3}$

\begin{tabular}{|c|r|r|r|r|}
\hline Groups & $\mathrm{N}$ & \multicolumn{1}{|c|}{ Mean } & Std. Deviation & Std. Error Mean \\
\hline $\mathrm{IV}_{2}$ & 10 & 34,890741 & 3,7408088 & 1,1829476 \\
$\mathrm{IV}_{3}$ & 10 & 31,986535 & 6,3983693 & 2,0233420 \\
\hline
\end{tabular}

\section{DISCUSSION AND CONCLUSIONS}

The results of the study described in this paper were:

- No quality differences between or within groups

- Significant difference in productivity between volume and variant products for $\mathrm{IV}_{2}$

- Significant difference in productivity between $\mathrm{IV}_{1}$ and $\mathrm{IV}_{2}$

- No significant difference in productivity between $\mathrm{IV}_{2}$ and $\mathrm{IV}_{3}$

- Variants disturb assembly of the following volume product

The results for the experiment were quite surprising, as a difference in quality between the independent variable states could not be found. Actually, each independent variable showed only 0-2 errors totally, which in no way indicates a difference in the quality parameter. This is easily attributed to a probable floor 
effect as a $r$ esult of the assembly probably being too easy. However, the productivity parameter did show differences between groups for hypothesis 1 but not for hypothesis 2 .

While the absence of quality differences between the groups can arguably be assigned to a floor effect, it is still unsure whether this is the whole truth. Since IV most likely did not hit a floor effect for the productivity parameter, it is somewhat uncertain whether it then can be affected by a floor effect for the quality parameter as quality and productivity very much go hand in hand. It could easily be argued that if a worker decides to work faster, this should have some kind of negative effect on quality. This issue is discussed in more depth in Thorvald et al. (2010). The quality issue will be investigated again in a follow up study to this one, again investigating the use of symbols and article numbers.

The significant difference, or absence thereof, between volume and variant products is to a large extent an enigma. As already mentioned there are several potential explanations to this such as floor effects and difficulties in understanding the instructions for $\mathrm{IV}_{1}$, but none of the data can support or reject any of these. It might be time to challenge the generally accepted opinion that variants take longer to assemble than do volume products.

Analysis of productivity between the three information interfaces did show significant differences between $\mathrm{IV}_{1}$ and $\mathrm{IV}_{2}$, as expected. It becomes evident that unsequenced, batched information is better than sequenced, un-batched information. This could be attributed to the minimalistic information design that it entails. Less information on the screen gives the worker a better overview of what is to be assembled and it is even plausible that the subject uses pattern recognition to help identify the type of product to assemble. The pattern recognition that humans are so adept at (Bouma \& De Voogd, 1974; Garzia \& Sesma, 1993; Rookes \& Willson, 2000) becomes much easier as the amount of data is reduced so drastically. A quick look at the unsequenced information can determine if everything is as it usually is. However, in the case of the sequenced information, a quick look will not be sufficient to determine what is to be assembled. In this case, subjects will most likely be forced to investigate the information source more thoroughly.

Productivity analysis of $\mathrm{IV}_{2}$ and $\mathrm{IV}_{3}$ did not show significant differences between the groups. However, post-hoc analysis of the data showed that the statistical power for the comparison of $\mathrm{IV}_{2}$ and $\mathrm{IV}_{3}$ was a mere 0,338 . This means that the experiment had only a $33,8 \%$ chance of finding a significant difference between the groups, had there been one. The low statistical power is thought to be a result of the relatively small sample size and a weaker than expected effect size. As mentioned above, the issue of using article numbers or using symbols to identify parts will be further investigated in coming studies.

To sum up, the experiment reached the following conclusions.

- The experiment hit a probable floor effect and thus no quality differences between the groups were found.

- No significant difference in productivity between volume and variant products could possibly challenge the view that variant products take 
longer to assemble.

- Significant difference in productivity between $\mathrm{IV}_{1}$ and $\mathrm{IV}_{2}$ but not between $\mathrm{IV}_{2}$ and $\mathrm{IV}_{3}$ indicates that the sequencing of information has greater impact on productivity than does the syntax used.

- This could also be attributed to a floor effect for $\mathrm{IV}_{2}$ and $\mathrm{IV}_{3}$.

The study reported in this paper has been both successful and unsuccessful. Successful in that it found productivity improvements when using an unsequenced, batched information interface and ramp up points after variant products. It was unsuccessful in that it could not give a conclusive answer to the use of article numbers or symbols in information presentation. This is largely due to the floor effect that is probably present in the experiment and the weak statistical power of the experiment. These two issues might have been nullified had the experiment had access to more subjects and a more difficult task. The subjects were provided through collaboration with a major manufacturer to ensure actual assembly workers in the experiment.

\section{REFERENCES}

Bouma, H. \& De Voogd, A. (1974). On the control of eye saccades in reading. Vision Research, 14 (4), 273-284.

Bäckstrand, G. (2010). Information Flow and Product Quality in Human Based Assembly. $\mathrm{PhD}$ Thesis. Loughborough University. Loughborough, UK. Wolfson School of Mechanical and Manufacturing Engineering.

Garzia, R. P. \& Sesma, M. (1993). Vision and reading. Journal of Opthalmic Vision Development (24), 4-51.

Rookes, P. \& Willson, J. (2000). Perception: Theory, Development and Organisation. London: Routledge.

Sawhney, R., Kannan, S. \& Li, X. (2009). Developing a value stream map to evaluate breakdown maintenance operations. International Journal of In dustrial and Systems Engineering, 4 (3), 229-240.

Thorvald, P. (2011). Presenting Information in Manual Assembly. PhD-Thesis. Loughborough University. Loughborough, UK. Wolfson School of M echanical and Manufacturing Engineering.

Thorvald, P., Brolin, A., Högberg, D. \& Case, K. (2010). Using Mobile Information Sources to Increase Productivity and Quality. In P roceedings of A pplied Human Factors and Ergonomics, Miami, Florida, July, 2010. 